\title{
Mortalidad atribuible a gripe en los períodos prevacunación y posvacunación en Argentina: estudio ecológico (2002-2016)
}

\author{
Elena B. Sarrouf, ${ }^{1}$ Reinaldo Souza-Santos ${ }^{2}$ y Oswaldo Gonçalves Cruz ${ }^{3}$
}

Forma de citar Sarrouf E, Souza-Santos R, Gonçalves Cruz O. Mortalidad atribuible a gripe en los períodos prevacunación y posvacunación en Argentina: estudio ecológico (2002-2016). Rev Panam Salud Publica. 2019;43:e15. https://doi.org/10.26633/RPSP.2019.15

RESUMEN Objetivos. Comparar las tasas de mortalidad por infección respiratoria aguda grave atribuible a gripe (IRAG) entre los períodos antes de la vacunación ( $A V$ ) y después de la vacunación $(D V)$, conocer la evolución histórica y estacionalidad de serie histórica entre 2002-2016 y estimar el riesgo de morir de niños entre 6 y 23 meses mediante el empleo de un modelo estadístico.

Métodos. Estudio de serie histórica con datos oficiales de mortalidad del informe estadístico de defunción. Se consideraron como IRAG los códigos de CIE-10 entre J09-18.9 y J22X. Se calcularon tasas brutas (TB) y ajustadas por edad (TAE) y se compararon los períodos $A V$ (2002-2009) y DV (2010-2016) con prueba de Х2. El mejor modelo estadístico fue el que comparó las defunciones por IRAG en niños durante el 2002 con otros años. Se analizaron los datos con programa $R$, nivel de significancia $P<0,05$.

Resultados. El 4,6\% (301 747) de las defunciones fueron por IRAG, con una edad mediana de 82 años, el porcentaje de fallecidos menores de dos años disminuyó en el período DV $(2,34 \%$ a $0,99 \%, P<0,05)$. Se presentó una marcada estacionalidad en invierno. Las TAE en gerontes pasaron de 259,8 AV a 328,6 por 100000 personas $D V(P<0,05)$. En los niños, la TB disminuyó de manera significativa, estimando que el riesgo de morir por IRAG, comparado con el año 2 002, fue significativamente menor durante 3 años $D V$.

Conclusiones. La disminución de la mortalidad por gripe en Argentina fue más evidente en niños, con un descenso estimado de 3,5 niños fallecidos por IRAG por mes.

Palabras clave Mortalidad; vacunas contra la gripe; gripe humana; enfermedades respiratorias; estudios de series temporales; Argentina.

La gripe es una enfermedad respiratoria aguda, autolimitada, causada por el virus influenza y posee una distribución mundial. El cuadro clínico varía desde un catarro de vías aéreas superiores hasta una neumonía que puede llevar a la

\footnotetext{
Departamento de Estadísticas de Salud de los Servicios, Dirección de Epidemiología, Ministerio de Salud Pública de la provincia de Tucumán, Argentina. Enviar correspondencia a Elena
} Sarrouf, elena_sarrouf@hotmail.com

\footnotetext{
2 Departamento de Endemias Samuel Pessoa, Escola Nacional de Saúde Pública, Fundação Oswaldo Cruz, Rio de Janeiro, Brasil.

3 Programa de Computação Científica, Fundação Oswaldo Cruz, Rio de Janeiro, Brasil.
}

muerte; se presenta en epidemias anuales de magnitud y gravedad variadas (1). Un reciente estudio realizado (2), estima que, en el año, se producen en todo el mundo entre 260000 y 650000 muertes por causas respiratorias; esto se calcula sin considerar las muertes causadas por la descompensación de enfermedades preexistentes, como las cardiovasculares. 
Las tasas más elevadas se presentan en la población que tiene más de 64 años de edad (2), los niños menores de dos años, embarazadas y personas con factores de riesgo (3-5).

Durante el invierno, en Argentina, las infecciones respiratorias agudas son uno de los motivos más frecuente de consulta e internación, y se encuentran entre las cinco primeras causas de muerte (6). Según las recomendaciones de la Organización Mundial de la Salud (OMS), Argentina inició la vacunación gratuita a los mayores de 64 años a partir del año 2003. En 2010 se realizó una Campaña Nacional, y desde 2011 fue incorporado al Calendario Nacional de Vacunación (7) para los niños de 6 a 23 meses, embarazadas, madres de niños menores de seis meses, personal de salud y personas entre 2 a 64 años con factores de riesgo. Según los datos publicados por el Programa Nacional de Inmunización del Ministerio de Salud Pública (PNI), durante la Campaña Nacional de Vacunación antigripal, se colocaron más de 8,7 millones de dosis de vacunas y se calcula una cobertura alrededor del $93 \%$ de la población estimada (8). De esta manera, la vacunación antigripal pasó a ser obligatoria y gratuita para toda la población, y debe ser reconocida por las obras sociales y servicios de salud prepagos.

Debido a que la decisión de incorporar una nueva vacuna al Calendario Nacional implica un elevado costo en la salud pública del país, nos propusimos evaluar el efecto de la vacunación antigripal en la población. Se plantearon los siguientes objetivos: comparar las tasas de mortalidad por infección respiratoria aguda grave atribuible a gripe (IRAG) entre los años previos y posteriores a la implementación de la vacunación; conocer la evolución histórica y la estacionalidad de las tasas de mortalidad por IRAG en Argentina entre los años 2002 y 2016; y estimar el riesgo de morir en niños entre 6 y 23 meses mediante un modelo estadístico para evaluar modificaciones de la mortalidad por IRAG.

\section{MATERIALES Y MÉTODOS}

Se realizó un estudio ecológico de serie de tiempo (ST) entre 2002 y 2016. Para evaluar el primer objetivo, el efecto de la vacunación de la población en la mortalidad por IRAG atribuible a gripe. Se realizaron estudios de series históricas de las tasas y se compararon las tasas entre los períodos antes y después de la vacunación antigripal. Para estimar el riesgo de morir en niños, se trabajó con modelos estadísticos hasta conseguir los mejores indicadores.

El trabajo fue aprobado por el comité de Ética e Investigación del Instituto Nacional de Epidemiología Dr. Juan H. Jara en noviembre de 2014. La base de datos de mortalidad no poseía datos de identificación de las personas, las otras bases empleadas fueron realizada a partir de datos poblacionales y de acceso gratuito.

Los datos fueron anónimos, procedentes de las siguientes bases:

Informe Estadístico de Defunción (IED): base de datos suministrado por la Dirección de Estadísticas e Información de Salud de Argentina (DEIS). Esta base se elabora a partir del IED cargado por cada provincia y luego compilados por la DEIS, según las normativas de la Ley Nacional 26413, el IED y el certificado de defunción (CD) que son llenados por el médico o personal de salud idóneo quienes certifican la muerte. Esto es obligatorio de realizar en todas las personas, ya sea que haya fallecido en una institución de salud, en el domicilio o en la vía pública. Los IED y CD son entregados al Registro Civil de las personas de cada jurisdicción. El personal que trabaja en Estadísticas Vitales de cada provincia, recolecta todos los IED para codificarlos y digitalizarlos en la oficina central de cada provincia. En la base de datos no figura información referida a la identificación del fallecido (número de documento de identidad, nombre y apellido). En el IED, se registran tres tipos de causas de muerte: directa, intermedia y básica. Desde el año 1997, se siguen las normas de las Clasificación Internacional de Enfermedades, décima revisión (CIE-10) para la codificación y selección de causa de muerte. En el análisis, se tuvo en cuenta la causa de muerte básica, definida como la enfermedad que inicia la cadena de acontecimientos patológicos que conducen a la muerte. Esta causa es la que considera el Ministerio de Salud Pública de Argentina (MSAL) para elaborar estadísticas de mortalidad atribuida a alguna enfermedad. Con base en las normativas de Vigilancia Epidemiológica del Ministerio de Salud, se consideraron muertes atribuidas a gripe los códigos de causa de muerte básica entre J09 y J18.9 (gripe y neumonías) y J22X (infección aguda sin especificar de las vías respiratorias inferiores).
Número de vacunas aplicadas y porcentaje de población vacunada o cobertura de la vacuna, por año y provincia: estos datos fueron proporcionados por el PNI y de datos oficiales publicados $(6,7)$.

Número de personas según edad y provincia obtenido de las publicaciones del Censo Nacional de las Personas realizado por Instituto Nacional de Estadísticas y Censos de Argentina (INDEC) para los años 2001 y 2010. Se utilizaron las proyecciones por provincia y grupo de edad publicadas por INDEC $(9,10)$. La población mensual se estimó mediante una proyección lineal entre el año en estudio y el posterior, según provincia y grupo de edad.

Población en niños 6 a 23 meses: a partir de la población censada en 2001 y en 2010, se obtuvo el total de niños de uno y dos años. Para estimar el grupo entre 6 a 12 meses de edad, se calculó la mitad de la población de niños menores de un año. Luego se sumaron a los niños que tenían un año, y entre los puntos 2001 y 2010 se realizó una proyección lineal simple entre 2001 y 2016.

Tipo de cepa viral circulante y cepa vacunal colocada: se realizó una base de dato con la información disponible en línea del MSAL y del Grupo Colaborativo GROG (11). Se consideró dicotómica y afirmativa cuando había por lo menos una coincidencia.

Se calcularon las tasas de mortalidad específica por IRAG utilizando la siguiente fórmula:

$$
M=\frac{f}{p} 100000
$$

Donde:

$M=$ tasa de mortalidad por IRAG.

$F=$ número de fallecidos por IRAG según edad, grupo de edad, provincia, mes y año.

$p=$ número de personas de igual edad, provincia, mes y año.

Se calcularon las siguientes tasas específicas de IRAG:

Tasa de mortalidad por periodo en la población global y en personas mayores de 64 años (gerontes). Se consideró como período anterior a la vacunación (AV) el comprendido entre los años 2002-2009 y como período posterior al 
inicio de la vacunación (DV) al que va desde 2010 hasta 2016. Se estratificó por provincias y se calculó la tasa de mortalidad bruta y la ajustada por edad (TAE) por método directo y comparación con la población mundial (12). Se estimaron los intervalos de confianza de $95 \%$ (IC95\%) de las tasas ajustadas y se compararon las tasas $\mathrm{AV}$ y DV con la prueba de la $\chi 2$; se consideró $P<0,05$ como el nivel de significancia.

Tasa de mortalidad por período de los niños entre 6 a 23 meses, calculada por cada $100 \mathbf{0 0 0}$. Se calcularon las tasas de mortalidad bruta de los períodos AV y DV de cada provincia y se las comparó con la prueba de la $\chi 2$, se consideró $P<0,05$ como el nivel de significancia.

Tasa de mortalidad mensual en la población global y en gerontes por cada 100000 habitantes y personas mayores de 64 años, respectivamente. Se estandarizó de manera indirecta por edad y mes de cada provincia y año de la serie de tiempo con la metodología propuesta por la OMS (12). Se utilizó como tasa estándar la estimada para el período $\mathrm{AV}$, calculada a partir de la media aritmética del período 2002-2009 por mes y población en estudio (numerador) y, en el denominador, la población de mitad del período (año 2005), según mes y población específica. Se realizó una estandarización indirecta debido al escaso número de casos mensuales. Se utilizaron los datos para realizar el análisis de ST.

Tasa bruta de mortalidad mensual de niños de 6 a 23 meses por provincia y total del país por cada 100000 niños de 6 a 23 meses.

Con las tasas mensuales brutas en niños y las TAE en la población global y gerontes, se realizaron los análisis de ST. Se descompuso y graficó la ST de cada uno de los grupos de edad (datos originales, data) y los componentes estacionales (seasonal), tendencia (trend) y residuos (remander) y se realizó la comparación relativa de sus componentes. Se utilizó el paquete Stats del programa estadístico $\mathrm{R}^{\circledR}$ (13).

Se realizaron gráficos de cajas del número de fallecidos por mes e histogramas de edad (por año de vida) entre los períodos AV y DV; en cada uno de ellos se calcularon las varianzas (Var) y las desviaciones estándares (DE). En las variables cuantitativas se sacaron sus medidas de tendencia central y se compararon con prueba de $\chi 2$; se consideró significativo un $P<0,05$.

Se seleccionó el grupo de niños entre 6 a 23 meses para evaluar el efecto en esta población específicamente, ya que se tuvo acceso al porcentaje de la población vacunada en cada año. Debido a que no hay en el país un patrón oro para la comparación de las tasas de mortalidad por mes y grupo de edad, fue preciso realizar modelos estadísticos para evaluar su comportamiento. Para ello, se estimó el efecto de la vacunación con un modelo estadístico de tipo modelos aditivos generalizado (GAM, por sus siglas en inglés) (14), que son una extensión de los modelos lineales generalizados (GLM, por sus siglas en inglés). La ventaja es que permiten la inclusión de una función de suavizamiento no paramétrica para una o más variables independientes. Ello es útil cuando no hay una relación lineal entre la variable independiente y la variable dependiente (15). Si bien este método es muy utilizado para el modelaje de datos que relacionan patologías respiratorias contaminantes del aire (16), recientemente se han se han publicado estudios relacionados con influenza $(15,17)$.
Se consideró:

$$
\boldsymbol{y}=\alpha+\sum_{i=1}^{n} f i(X i)+\varepsilon
$$

Donde:

$Y=$ es la variable respuesta (niños fallecidos por IRAG).

$X_{i}=$ son las variables predictoras: mes, año, dosis de vacunas en el año, porcentaje de población vacunada, período DV y coincidencia entre la cepa de la vacuna y la cepa viral en el año (variable dicotómica).

$\alpha=$ es una constante.

$\varepsilon=$ es el error.

$f_{i}=$ se estiman mediante el uso de suavizadores.

Se utilizó una distribución binomial negativa debido a la diferencia entre la varianza y la media de los datos. Los grados de libertad se asignaron de manera automática, considerando el mejor valor posible. Se utilizaron suavizadores de tipo spline (s) para estimar funciones no paramétricas. El procedimiento del GAM contempló el valor de significancia $(P<0,05)$ y se seleccionó el modelo teniendo en cuenta el menor valor de máxima verosimilitud restringida (REML, por sus siglas en inglés) y el principio de parsimonia o navaja de Ockham. El modelo elegido arrojó un REML de 538,06 y una

FIGURA 1. Histograma comparativo entre el período antes de la vacunación y el período después de la vacunación de muertes por IRAG, Argentina

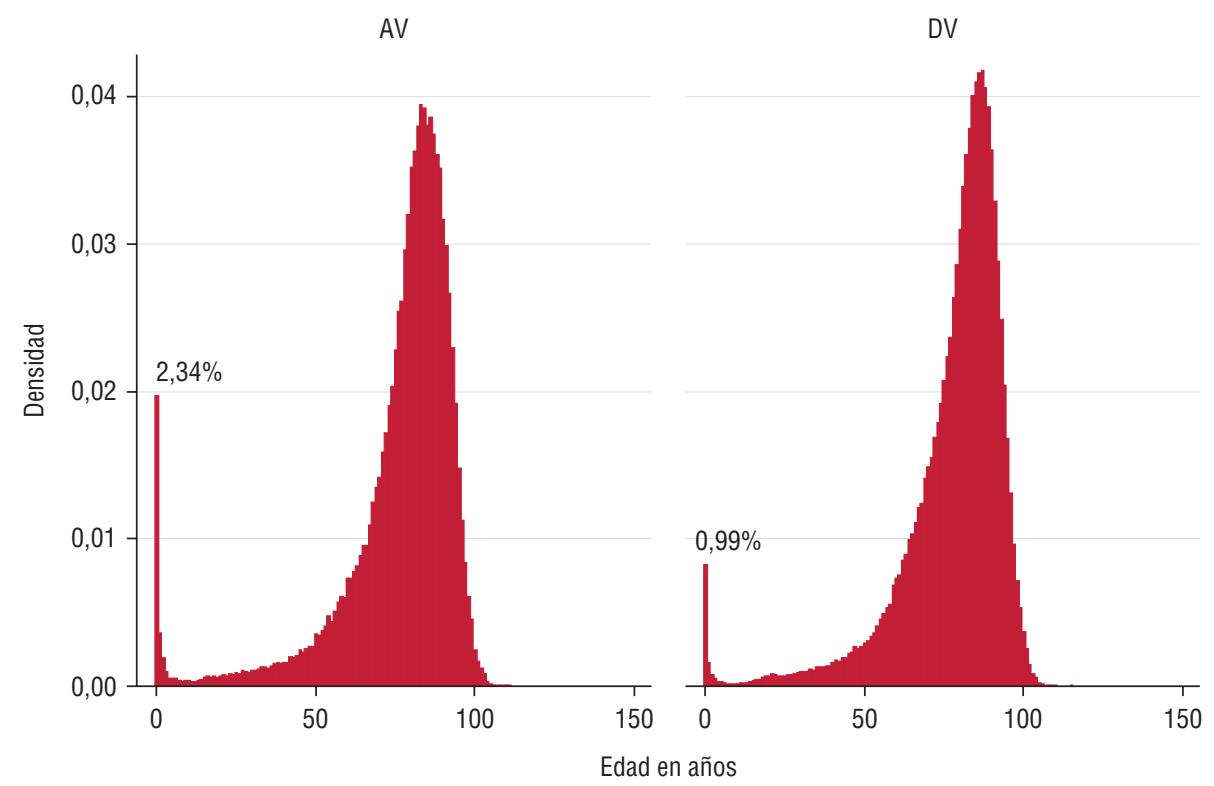

AV (período antes de la vacunación, 2002-2009, $n=126$ 701); DV (período después de la vacunación, 2010-2016, $n=174285$ casos)

Fuente: elaboración propia con datos oficiales de mortalidad obtenidos de la Dirección de Estadísticas e Información de Salud de Argentina (DEIS). 


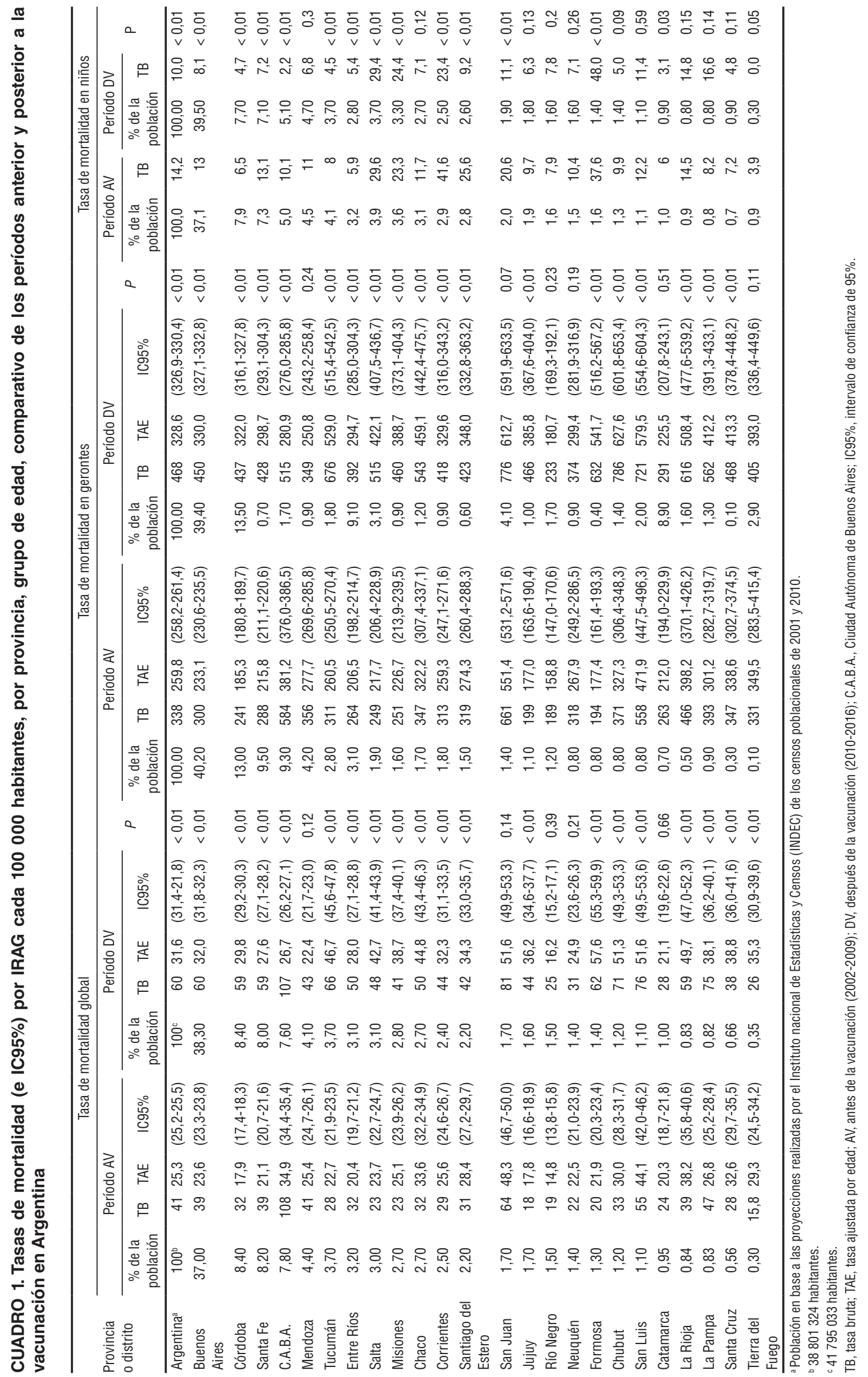


FIGURA 2. Gráfico de caja de los casos de IRAG según el mes de defunción y ST de la tasa de mortalidad (por cada 100 000 ) por IRAG, según grupo en Argentina (2002-2016)
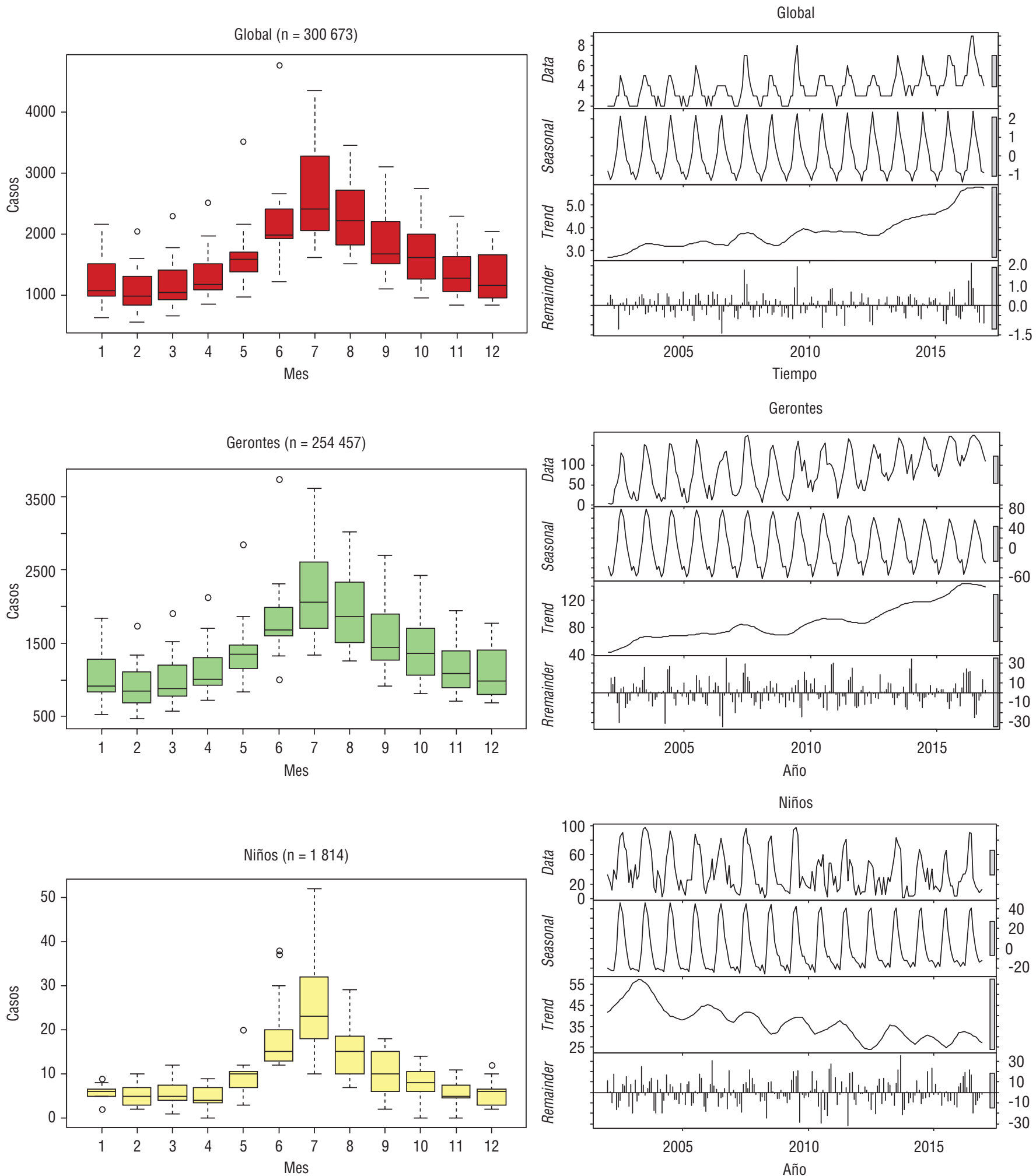

Fuente: elaboración propia a partir de datos de mortalidad de la Dirección de Estadísticas e Información de Salud. 
varianza explicada de $60,3 \%$, relacionando en función del mes, el número de niños fallecidos, la población y compara cada año con los valores del año 2002 (intercepto): Caso $\sim \mathrm{f}($ mes $)+$ factor $($ año $)+$ offset $=$
$\log (\mathrm{pn})$

Donde:

Caso: el número de niños 6 a 23 meses de edad, fallecidos por IRAG. f (mes): al mes de la defunción. factor (año): a cada año.

offset $=\log (p n)$ : enlace de la función del logaritmo de la población de niños.

Los datos se analizaron con el programa $\mathrm{R}^{\circledR}$ y el paquete mgcv (18). Para el modelo seleccionado se calculó el riesgo relativo (RR) y el IC95\% comparando el riesgo de morir en el año 2002 con los otros años y comparando los meses del año 2002 con los meses de los otros años. Se calculó la media aritmética de los períodos AV y DV de los fallecidos por mes en el modelo planteado.

\section{RESULTADOS}

De las 4691147 personas fallecidas entre los años 2002 y 2016, 15,2\% fue por patología respiratoria (J00-J98.9) y el 6,4\% (301 747) por IRAG; en especial por neumonía (J18), que representó 92,3\% (31 219) de las IRAG denunciadas. El promedio anual de personas fallecidas por IRAG tuvo un incremento entre los períodos AV y DV, con 15869 y 24971 casos, respectivamente. La media de edad fue de 78 años, la mediana fue de 82 años y la mitad de los fallecidos tenía entre 72 y 88 años. En el histograma de edad se pudo observar una mayor concentración de datos en el período DV (edad del período AV: Var 372,5 y DE 19,3; edad período DV: Var 275,9 y DE 16,6). En comparación con el histograma del período $\mathrm{AV}$, hubo una disminución del porcentaje de los casos en menores de dos años $(P<0,05)$ (figura 1), que pasó de representar el $2,34 \%$ del total de fallecidos por IRAG en el período AV (126 701) a ser el 0,99\% de los fallecidos en el período DV (174 285).

Las TAE de la población total (tasas globales) y en personas mayores de 64 años (tasas en gerontes) aumentaron de manera significativa excepto en la Ciudad Autónoma de Buenos Aires (C.A.B.A.) (cuadro 1). La tasa bruta de mortalidad en niños entre 6 a 23 meses evidenció una disminución significativa en 14 de las 24 jurisdicciones (provincias y C.A.B.A.) del país (cuadro 1).

La descomposición de la ST de las tasas mensuales mostró, en los tres grupos estudiados, un marcado comportamiento estacional, con picos en el mes de julio (figura 2). La tendencia de la mortalidad en los niños parece descender a partir del año 2 004; mientras que en los adultos mayores y población global parece ascender en los últimos años. Luego de eliminado el efecto de la tendencia y estacionalidad, se evidenció un pico de la mortalidad en 2009 en los niños y en 2016 en los adultos mayores (figura 2).

Para estimar el riesgo de morir en niños entre 6 y 23 meses se realizaron varios modelos estadísticos GAM. Si bien se incorporaron diferentes variables como el número y el porcentaje de vacunación y la coincidencia de la cepa viral y la de la vacuna del mismo año, el mejor modelo fue el más simple. Este comparó el riesgo de morir en el año 2002 con los otros años en el grupo de niños entre 6 a 23 meses, y fue significativamente menor en un año AV, el 2008, y en cuatro años del período DV (2010, 2012, 2014 y 2015) (cuadro 2). En base a los valores obtenidos del modelo, se estimó que el

CUADRO 2. Número de casos y riesgo de fallecer por IRAG en niños de 6 a 23 meses comparando el riesgo en el año 2002 y cobertura de vacunación $(n=1814)$

\begin{tabular}{|c|c|c|c|c|c|c|c|c|}
\hline \multirow{3}{*}{ Aก̃o } & \multirow{3}{*}{$\begin{array}{l}\text { Número } \\
\text { de casos }\end{array}$} & \multirow{3}{*}{$\begin{array}{l}\text { Población } \\
\text { (hab.) }^{\text {a }}\end{array}$} & \multirow{3}{*}{$\mathrm{RR}$} & \multirow{3}{*}{ IC95\% } & \multicolumn{4}{|c|}{ Población vacunada } \\
\hline & & & & & \multicolumn{2}{|c|}{ Primera dosis } & \multicolumn{2}{|c|}{ Segunda dosis } \\
\hline & & & & & $\%$ & $\begin{array}{l}\text { Número } \\
\text { de dosis }{ }^{\mathrm{b}}\end{array}$ & $\%$ & $\begin{array}{l}\text { Número } \\
\text { de dosis }\end{array}$ \\
\hline 2002 & 141 & 971730 & \multicolumn{2}{|c|}{ (Intercepto) } & \multicolumn{2}{|c|}{$N A$} & \multicolumn{2}{|c|}{ NA } \\
\hline 2003 & 208 & 977113 & $1,3^{c}$ & $0,9-1,9$ & \multicolumn{2}{|c|}{ NA } & \multicolumn{2}{|c|}{ NA } \\
\hline 2004 & 132 & 982496 & $0,9^{c}$ & $0,6-1,3$ & \multicolumn{2}{|c|}{ NA } & \multicolumn{2}{|c|}{ NA } \\
\hline 2005 & 120 & 987878 & $0,8^{c}$ & $0,6-1,2$ & \multicolumn{2}{|c|}{ NA } & \multicolumn{2}{|c|}{ NA } \\
\hline 2006 & 126 & 993261 & $0,9^{c}$ & $0,6-1,3$ & \multicolumn{2}{|c|}{ NA } & \multicolumn{2}{|c|}{ NA } \\
\hline 2007 & 141 & 998644 & $0,9^{c}$ & $0,6-1,3$ & \multicolumn{2}{|c|}{ NA } & \multicolumn{2}{|c|}{ NA } \\
\hline 2008 & 98 & 1004027 & $0,6^{\mathrm{d}}$ & $0,4-0,9$ & \multicolumn{2}{|c|}{ NA } & \multicolumn{2}{|c|}{ NA } \\
\hline 2009 & 159 & 1009409 & $0,9^{c}$ & $0,7-1,4$ & \multicolumn{2}{|c|}{ NA } & \multicolumn{2}{|c|}{ NA } \\
\hline 2010 & 98 & 1014792 & $0,7^{c}$ & $0,5-1,0$ & 86,7 & 949075 & 57,7 & 629179 \\
\hline 2011 & 107 & 1020175 & $0,7^{c}$ & $0,5-1,0$ & 73,0 & 792877 & 36,4 & 395569 \\
\hline 2012 & 78 & 1025557 & $0,5^{\mathrm{e}}$ & $0,4-0,8$ & 75,7 & 811843 & 58,2 & 624164 \\
\hline 2013 & 109 & 1030940 & $0,7^{c}$ & $0,5-1,0$ & 78,1 & 848663 & 46,0 & 511567 \\
\hline 2014 & 89 & 1036323 & $0,6^{e}$ & $0,4-0,9$ & 73,8 & 801219 & 50,4 & 547174 \\
\hline 2015 & 79 & 1041706 & $0,5^{\mathrm{e}}$ & $0,3-0,8$ & 81,3 & 882644 & 60,3 & 654655 \\
\hline 2016 & 129 & 1047088 & $0,8^{c}$ & $0,6-1,2$ & 78,6 & 853331 & 59,9 & 650312 \\
\hline
\end{tabular}

a Estimaciones con base en el Censo 2001 y 2010.

${ }^{b}$ Datos publicados por el Ministerio de Salud de la Nación Argentina.

c $P>0,05$.

d $P<0,05$.

e $P<0,01$.

$\mathrm{RR}$, riesgo relativo; IC95\%, intervalo de confianza de 95\%; NA, no aplica. promedio mensual de niños fallecidos por IRAG disminuyó alrededor de 3,5 entre el período AV y DV, pasó de 11,89 a 8,19 defunciones mensuales, respectivamente (figura 3A). Se analizó el parámetro estacional del modelo planteado que comparó los meses de 2002 con los otros años; se observó que el RR de morir por IRAG en los niños de 6 a 23 meses fue de 0,46 (IC95\%: 0,36-0,57) durante abril. A fines de junio el riesgo llegó a casi el doble ( $R R=2,7$; IC95\%: 2,3-3,3) (figura 3B). El RR de morir por IRAG en este grupo de edad se mostró aumentado entre finales de mayo y julio, en comparación con el riesgo durante el año 2002.

\section{DISCUSIÓN}

En la ST estudiada, la causa de muerte atribuible a IRAG más frecuente fue la neumonía por microorganismo no especificado (J18 de CIE-10), al igual que en otros estudios realizados en el país (19). Las tasas de mortalidad por IRAG mostraron un ascenso entre el período AV y DV en la población total de Argentina y en los mayores de 64 años. Esto coincide con la tendencia en ascenso observada en la descomposición de la serie histórica de los 15 años estudiados. Las tasas observadas en los mayores de 64 años 
fueron similares a las encontradas en otros estudios en el país (20) y en Brasil (21), pero mayores a las reportadas en Chile y otros países del hemisferio sur (22). Una revisión sistemática del Grupo Colaborativo Cochrane (23) estimó que los adultos mayores vacunados podrían tener un menor riesgo de enfermarse de

\section{FIGURA 3. Modelo estadístico planteado}

A. Serie de tiempo del número de niños fallecidos, según el mes y el año en Argentina $(2002-2016)(n=1814)$

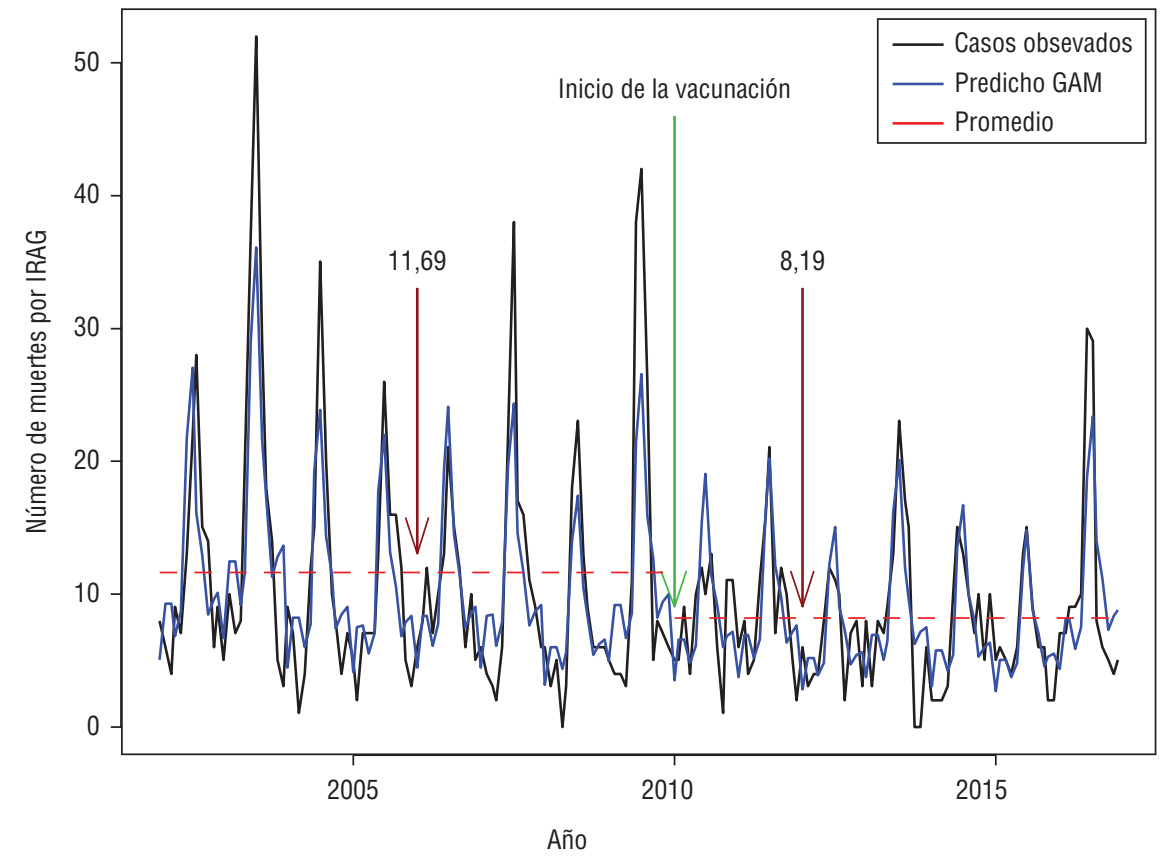

B. Riesgo relativo de fallecer, según mes comparado con 2002

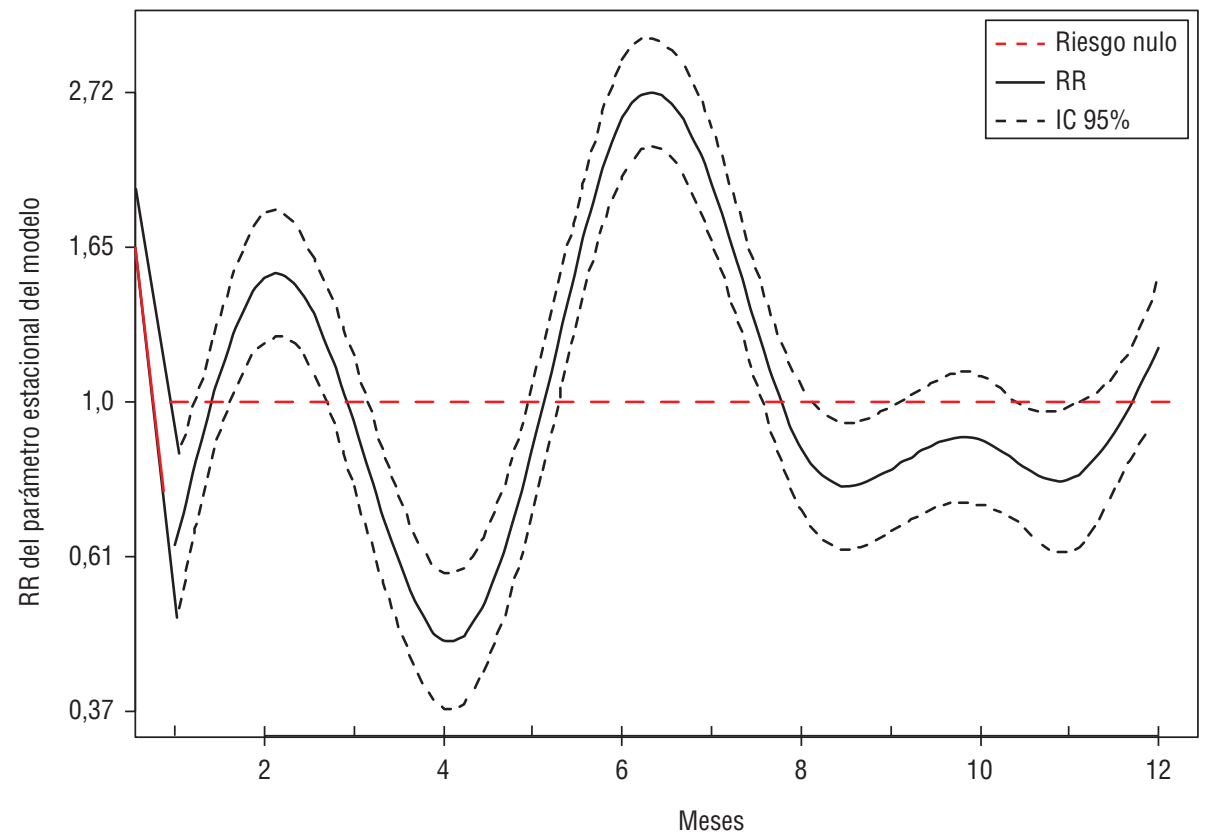

Fuente: elaboración propia a partir de datos presentados gripe en la misma estación comparados con aquellos no vacunados (RR: 0,42; IC95\%: 0,27-0,66). No obstante, el estudio no pudo estimar el efecto de la vacunación en la mortalidad debido a la poca información en los estudios seleccionados. En Argentina, no hay una cifra oficial del porcentaje de población de

mayores de 64 años vacunados, debido a que se realiza en el sector público, privado y de seguridad social. Recién en el año 2013 se realizó la primera encuesta poblacional sobre la cobertura de vacunación en el país (24), en la que se reportó que se había vacunado 51,6\% (IC95\%: $49,6-53,6)$ del total de personas encuestadas y 55,6\% (IC95\%: 52,8-58,3) de las personas mayores de 64 años sin comorbilidad. Si bien la vacunación antigripal en mayores de 64 años se realiza de forma obligatoria desde el año 2003, este estudio pretendió mostrar el posible efecto de la disminución de los susceptibles con la inmunidad de grupo.

Con respecto a las tasas de mortalidad bruta en niños entre 6 a 23 meses, se observó una disminución en la mayoría de las provincias entre el periodo AV y DV, similar a lo observado en una revisión sistemática del grupo Cochrane (25).

Los modelos estadísticos semiparamétricos, como el GAM, pueden reemplazar a los modelos armónicos estacionales convencionales para estimar las enfermedades respiratorias y la gripe. Un estudio realizado en Australia (15) modeló con GAM para evaluar la mortalidad atribuible a influenza en una serie histórica, como el presente trabajo.

En el análisis de la ST y en el modelo GAM realizado, el componente estacional fue bien marcado, con un claro incremento de la mortalidad entre junio y setiembre, con pico en julio, similar al comportamiento observado en otros estudios (26-29).

Este estudio tiene limitaciones, sobre todo por ser un estudio ecológico y la poca información pública disponible en el país. En primer lugar, se utilizó un grupo de enfermedades que se le puede atribuir a gripe, pero no se confirmó la etiología por virus influenza de las IRAG, que pueden haber sido causadas por otros virus respiratorios. En el país, la vigilancia de virus respiratorios se realiza en algunas provincias, en especial en la población pediátrica. Hay estudios que muestran que el comportamiento es diferente en niños y en mayores de 64 años (30). En segundo lugar, la fuente de datos fue el IED. Si bien se trata de datos oficiales y denunciados por profesionales, puede tener un margen de error en el registro, con sobreestimación o subestimación de las muertes por gripe. Otras medidas sanitarias no pudieron ser evaluadas, como el lavado de manos, la mejora en la atención de los pacientes, 
el inicio temprano de la medicación antigripal, la educación sanitaria, el avance tecnológico y el terapéutico durante los 15 años estudiados, la incorporación de la vacuna antineumocócica en el año 2012, las temperaturas en las diferentes regiones del país y la fecha de inicio de la vacunación con las nuevas cepas cada año.

\section{Conclusiones}

Las tasas de mortalidad por IRAG se mantuvieron similares en los adultos mayores de 64 años y en la población general entre los períodos AV y DV. En cambio, en los niños, se observó un claro descenso. Se estima que los niños entre 6 a 23 meses tuvieron menor riesgo de morir por IRAG en los tres años posteriores a la vacunación, en comparación con el año 2002.

1. World Health Organization (WHO). Influenza virus infections humans, 2018. Disponible en: http://www.who.int/influenza/human_animal_interface/virology_laboratories_and_vaccines/influenza virus_infections_humans_Oct_18.pdf?ua =1

2. Iuliano AD, Roguski KM, Chang $\mathrm{HH}$, Muscatello DJ, Palekar R, Tempia S, et al. Estimates of global seasonal influenza-associated respiratory mortality: a modelling study. Lancet Respir Med. 2017;391(10127): 1285-300. Disponible en: https://journals. plos.org/plosone/article?id=10.1371/ journal.pone.0064734 Acceso el 17 de agosto de 2018.

3. Martín V, Castilla J, Godoy P, DelgadoRodríguez M, Soldevila N, Fernández-Villa $\mathrm{T}$, et al. Índice de masa corporal elevado como factor de riesgo de hospitalización por gripe: estudio de casos y controles. Arch Bronconeumol. 2016;52(6):299-307. Disponible en: https://www.archbronconeumol.org/es-indice-masa-corporal-elevado-como-articulo-S0300289615004652 Acceso el 10 de agosto de 2018.

4. Sarrouf EB, Cuezzo MR, Nuñez Mrad PR, Hilal FA, Ramírez FA, Alcaraz G, et al. Comportamiento de la pandemia por gripe A de 2009 en niños menores de cinco años de la Provincia de Tucumán, Argentina. Rev Argent. 2010;1(4):40-2.

5. Dwyer DE, Lynfield R, Losso MH, Davey RT, Cozzi-Lepri A, Wentworth D, et al. Comparison of the outcomes of individuals with medically attended influenza $A$ and $B$ virus infections enrolled in 2 international cohort studies over a 6-year period: 2009-2015. Open Forum Infect Dis. 2017;4(4). Disponible en: https://academic.oup.com/ofid/article/doi/10.1093/ ofid/ofx212/4372244 Acceso el 3 de octubre de 2018 .
Si bien la vacuna antigripal está disponible durante todo el año, la marcada estacionalidad que presenta la enfermedad hace necesario planificar la distribución de las partidas de la nueva vacuna desde principios del año, así podría estar disponible unos meses antes del ascenso de la epidemia anual.

Este trabajo pretende delinear un panorama general del efecto de la vacunación antigripal en la población luego de la implementación de una medida poblacional de salud pública. Esto debe ir acompañado de la educación de la población y profesionales de la salud, del avance tecnológico en la atención y tratamiento y un buen sistema de vigilancia epidemiológica que genere datos para la toma de decisiones políticas.

Agradecimientos. Los autores agradecen la colaboración del Programa

\section{REFERENCIAS}

6. Ministerio de Salud de la República Argentina. Lineamientos técnicos y manual del vacunador. Influenza o gripe. Vacuna antigripal 2017. Disponible en: http:// www.msal.gob.ar/images/stories/bes/ graficos/0000000950cnt-2017-04_lineamientos-gripe_2017.pdf Acceso el 10 de octubre de 2018.

7. Ministerio de Salud de la República Argentina. Lineamientos técnicos para la vacunación antigripal, Argentina, 2014. Disponible en: http://www.msal.gov.ar/ images/stories/bes/graficos / 0000000 450cnt-2014-03_lineamientos-gripe-2014final.pdf

8. Gripe A (H1N1) y vacunación. Folia Doc. 2010. Disponible en: http://www.fundacionfemeba.org.ar / farmacologia / folia-doc-gripe-h1n1-y-vacunaci\%C3\%B3n

9. Instituto Nacional de Estadísticas y Censo Argentina (INDEC). Proyecciones provinciales de población 2010 a 2 040, por sexo y grupo de edad. Disponible en: https:// www.indec.gob.ar/nivel3_default.asp? id_tema_1=2\&id_tema_2 $=24$

10. Dirección Nacional de Estadísticas de Salud. Proyecciones poblacionales por provincia según grupo de edad y sexo 2001 a 2 010. INDEC Censo 2001. Disponible en: https: / / www.indec.gov.ar/cgibin / Rp WebEngine.exe/nivel3_default.asp?id_ tema_1=2\&id_tema_2=24

11. Grupo Colaborativo de Vigilancia Epidemiológica de gripe y otras virosis respiratorias. Boletín GROG 2002 al 2007. Buenos Aires, Argentina; 2008. Disponible en: http:/ / www.grog-argentina.org

12. Organización Panamericana de la Salud (OPS). Módulos de principios de epidemiología para el control de enfermedades: investigación epidemiológica de campo: aplicación al estudio de brotes. Washington,
Nacional de Inmunización, de la Dirección de Estadísticas e Información en Salud de Argentina (DEIS) y de la Dirección de Epidemiología de la provincia de Tucumán, quienes suministraron las bases de datos para poder realizar los análisis. También quieren destacar la colaboración del Ministerio de Salud Pública de Tucumán y a la Fundación Fio-Cruz (Rio de Janeiro, Brasil), por su la asesoría técnica y metodológica, y a Enrique U. López por su colaboración para la realización de la investigación.

Conflicto de intereses. Ninguno declarado por los autores.

Declaración. Las opiniones expresadas en este manuscrito son responsabilidad del autor y no reflejan necesariamente los criterios ni la política de la RPSP/ PAJPH y / o de la OPS.
D.C: OPS; 2002. Disponible en: http:// www.paho.org $/ \mathrm{col} /$ index.php?option= com_docman\&task=doc_download\&gi$\mathrm{d}=855 \&$ Itemid $=$

13. Cleaveland RB, Cleaveland WS, McRae JE, Terpenning I. STL: a seasonal-trend decomposition procedure based on Loess. J Off Stat. 1990;6(1):3-73.

14. Ministerio da Saúde, Fundação Oswaldo Cruz. Introdução à Estatística Espacial para a Saúde Pública. Brasilia; 2007. Disponible en: http://www.escoladesaude.pr.gov.br/ arquivos/File/textos_curso_vigilancia/capacitacao_e_atualizacao_em_geoprocessamento_em_saude_3.pdf

15. Muscatello DJ, Newall AT, Dwyer DE, MacIntyre CR. Mortality attributable to seasonal and pandemic influenza, Australia, 2003 to 2009, using a novel time series smoothing approach. Cowling BJ, editor. PLoS ONE. 20138(6):e64734.. Disponible en: http:/ / dx.plos.org/10.1371/journal. pone.0064734

16. Dominici F, McDermott A, Zeger SL, Samet JM. On the use of generalized additive models in time-series studies of air pollution and health. Am J Epidemiol. 2002;156(3): 193-203. Disponible en: http://dx.plos. org/10.1371/journal.pone.0064734 Acceso el 10 de setiembre de 2018.

17. Li T, Cheng Z, Zhang L. Developing a novel parameter estimation method for agent-based model in immune system simulation under the framework of history matching: a case study on influenza a virus infection. Int J Mol Sci.2017;18(12):2592. Disponible en: http:/ / www.mdpi.com/1422-0067/ 18/12/2592 Acceso el 10 de setiembre de 2018.

18. Wood S, Wood MS. Mixed GAM computation vehicle with GCV/AIC/REML smoothness estimation. 2015. Disponible 
en: http:/ / cran.stat.auckland.ac.nz/web/ packages $/ \mathrm{mgcv} / \mathrm{mgcv}$.pdf

19. Gentile A, Lucion MF, del Valle Juarez M, Martinez AC, Romanin V, Bakir J, et al. Influenza virus: 16 years' experience of clinical epidemiologic patterns and associated infection factors in hospitalized children in Argentina. Lin B, editor. Plos ONE.2018;13(3):e0195135. Disponible en: http: / / dx.plos.org/10.1371/journal. pone.0195135 Acceso el 10 de setiembre de 2018.

20. Azziz-Baumgartner E, Cabrera AM, Cheng P-Y, Garcia E, Kusznierz G, Calli R, et al. Incidence of influenza-associated mortality and hospitalizations in Argentina during 2002-2009: Influenza mortality and hospitalizations. Influenza Other Respir Viruses. 2013;7(5):710-7.

21. Marinho de Souza M de F, Widdowson M-A, Alencar AP, Gawryszewski VP, AzizBaumgartner E, Palekar R, et al. Trends in mortality from respiratory disease in Latin America since 1998 and the impact of the 2009 influenza pandemic. Bull World Health Organ. 2013;91(7):525-32. Disponible en: http://www.who.int/entity/bulletin/ volumes/91/7/12-116871.pdf Acceso el 10 de setiembre de 2018.

22. Sotomayor V, Fasce RA, Vergara N, De la Fuente F, Loayza S, Palekar R. Estimating the burden of influenza-associated hospitalizations and deaths in Chile during 2012-2014. Influenza Other Respir Viruses. 2018;12(1):138-45. Disponible en: http:// doi.wiley.com/10.1111/irv.12502 Acceso el 16 de octubre de 2018.

23. Demicheli V, Jefferson T, Di Pietrantonj C, Ferroni E, Thorning S, Thomas RE, et al.
Vaccines for preventing influenza in the elderly. Cochrane Acute Respiratory Infections Group, editor. Cochrane Database Syst Rev. 2018. Disponible en: http://doi.wiley.com/10.1002/14651858. CD004876.pub4

24. Vizzotti C, Katz N, Stecher D, Aquino A, Juárez MDV, Urueña A. Evaluación del uso en adultos de cuatro vacunas: una encuesta poblacional en Argentina. Med B Aires. 2018;78(2):76-82.

25. Jefferson T, Rivetti A, Di Pietrantonj C, Demicheli V. Vacunas para prevenir la influenza en niños sanos (revisión sistemática). Cochrane Database Syst Rev. 2018; CD004879(2):31-31.Disponible en: https:// www.cochranelibrary.com / cds r / doi/10.1002/14651858.CD004879.pub5/ full?highlightAbstract=influenza\&highlightAbstract=influenz\&highlightAbstrac$\mathrm{t}=$ vaccin\&highlightAbstract=vaccine\&highlightAbstract=influnza Acceso el 1 de febrero de 2018.

26. Baumeister E, Duque J, Varela T, Palekar $\mathrm{R}$, Couto P, Savy V, et al. Timing of respiratory syncytial virus and influenza epidemic activity in five regions of Argentina, 2007-2016. Influenza Other Respir Viruses. Disponible en: http://doi.wiley.com/10. 1111/irv.12596 Acceso el 3 de octubre de 2018.

27. Caini S, Alonso WJ, Balmaseda A, Bruno A, Bustos P, Castillo L, et al. Characteristics of seasonal influenza A and B in Latin America: Influenza surveillance data from ten countries. Shaman J, editor. Plos ONE. 2017;12(3):e0174592. Disponible en: 10.1371/journal.pone.0174592 Acceso el 5 de octubre de 2018 .
28. Caini S, Andrade W, Badur S, Balmaseda A Barakat A, Bella A, et al. Temporal patterns of influenza A and B in tropical and temperate countries: what are the lessons for influenza vaccination? Schanzer DL, editor. Plos ONE. 2016;11(3):e0152310. Disponible en: http://dx.plos.org/10.1371/journal. pone.0152310 Acceso el 5 de octubre de 2018.

29. Woyessa AB, Mengesha M, Belay D, Tayachew A, Ayele W, Beyene B, et al. Epidemiology of influenza in Ethiopia: findings from influenza sentinel surveillance and respiratory infection outbreak investigations, 2009-2015. BMC Infect Dis. 2018;18(1). Disponible en: https:// bmcinfectdis.biomedcentral.com/ articles / 10.1186/s12879-018-3365-5 Acceso el 3 de octubre de 2018.

30. Global Influenza B Study group, Caini S Spreeuwenberg P, Kusznierz GF, Rudi JM, Owen R, et al. Distribution of influenza virus type by age using case-based global surveillance data from twenty-nine countries, 1999-2014. BMC Infect Dis. 2018; 18(1). Disponible en: https://bmcinfectdis.biomedcentral.com/articles/10.1186/ s12879-018-3181-y Acceso el 3 de octubre de 2018.

Manuscrito recibido el 5 de junio de 2018. Aceptado para su publicación, tras revisión, el 25 de octubre de 2018. 
ABSTRACT

Mortality attributable to influenza in pre-vaccination and post-vaccination periods in Argentina: an ecological study (2002-2016)

Objectives. Compare mortality from severe acute respiratory infection (SARI) attributable to influenza between pre-vaccination (pre-V) and post-vaccination (post-V) periods, to determine the historical evolution and seasonality of time series between 2002 and 2016, and to estimate the risk of death in children between 6 and 23 months of age, using a statistical model.

Methods. Time-series study using official mortality data from the official statistical database on deaths. ICD-10 codes between J09-18.9 and J22X were considered to represent SARI. Crude rates and age-adjusted rates (AAR) were calculated, and pre-V (2002-2009) and post-V (2010-2016) periods were compared using the chi-squared $(\chi 2)$ test. The best statistical model was the one that compared deaths from SARI in children during 2002 with other years. The data were analyzed with $\mathrm{R}$ programming $(p<0.05)$.

Results. $4.6 \%$ of deaths $(301,747)$ were from SARI, with a median age of 82 years. The percentage of deaths under age 2 declined in the post-V period (from $2.34 \%$ to $0.99 \%, p<0.05)$. Marked seasonality was observed in winter. The AAR in persons over age 64 rose from 259.8 per 100,000 population (pre-V) to 328.6 (post-V) $(p<0.05)$. In children, the crude rate dropped significantly. Compared with the year 2002, there was a significantly lower estimated risk of dying from SARI during the three years post-V.

Conclusions. The reduction in mortality from influenza in Argentina was more pronounced in children, with an estimated 3.5 fewer child deaths from SARI per month.

Keywords Mortality; influenza vaccines; influenza, human; respiratory tract diseases; time series studies; Argentina.

RESUMO

Mortalidade atribuível à gripe
no período pré-vacinal e pós-
vacinal na Argentina: estudo
ecológico (2002-2016)

Palavras-chave
Objetivos. Comparar o índice de mortalidade por infecção respiratória aguda grave atribuível à gripe nos períodos pré-vacinal e pós-vacinal, conhecer a evolução temporal e a sazonalidade da série temporal entre 2002 e 2016 e estimar o risco de morte em crianças entre 6 e 23 meses de idade com o uso de um modelo estatístico.

Métodos. Estudo de série histórica com base em dados oficiais de mortalidade obtidos do informe estatístico de óbitos. Os códigos da CID-10 entre J09-18.9 e J22X foram considerados como sendo infecção respiratória aguda grave atribuível à gripe. Foram calculados os índices de mortalidade brutos e ajustados por idade e feita uma comparação entre os períodos pré-vacinal (2002-2009) e pós-vacinal (2010-2016) com o teste de $\chi 2$. O melhor modelo estatístico foi o que comparou os índices de mortalidade por infecção respiratória aguda grave atribuível à gripe em crianças em 2002 com os outros anos. Os dados foram analisados com o programa R, a um nível de significância $P<0,05$.

Resultados. Observou-se que 4,6\% (301.747) das mortes foram por infecção respiratória aguda grave atribuível à gripe, com idade mediana de 82 anos. Houve queda no percentual de mortes em crianças menores de dois anos no período pós-vacinal $(2,34 \%$ a $0,99 \%, P<0,05)$. Verificou-se uma acentuada sazonalidade no inverno. Os índices ajustados por idade em idosos variaram entre 259,8 por 100 mil no período pré-vacinal e 328,6 por 100 mil no período pós-vacinal $(P<0,05)$. Nas crianças, houve queda significativa nos índices brutos, estimando-se um risco significativamente menor de morte por infecção respiratória aguda grave atribuível à gripe nos 3 anos pós-vacinais em comparação a 2002.

Conclusões. A redução da mortalidade pela gripe na Argentina foi mais evidente em crianças, com uma redução estimada de 3,5 mortes por mês.

Mortalidade; vacinas contra influenza; influenza humana; doenças respiratórias; estudos de séries temporais; Argentina. 